\title{
Special issue on 2020 international conference on machine learning and big data analytics for loT security and privacy (SPIoT-2020)
}

\author{
Jinghua Zhao' ${ }^{1}$ Qingyuan Zhou ${ }^{2}$ \\ Published online: 25 February 2021 \\ (C) The Author(s), under exclusive licence to Springer-Verlag London Ltd. part of Springer Nature 2021
}

This special issue includes selected papers (with no less than $60 \%$ new content of the journal version) from the 2020 International Conference on Machine Learning and Big Data Analytics for IoT Security and Privacy (SPIoT2020), November 6 and 8, 2020, Shanghai, China as well as an open call. The submitted manuscripts were reviewed by experts from both academia and industry. After two rounds of reviewing, the highest quality manuscripts were accepted for this special issue. This special issue will be published by Neural Computing and Applications as special issues. Totally, 19 papers are suggested to EiC for acceptance. The selected papers are summarized as follows.

Huang and Zhu [1] study the practicability of statistical protocol recognition methods in network environment and to develop new solutions based on the existing protocol recognition methods. Zhang and Zhang [2] discuss the research of the Top- $\mathrm{N}$ recommendation algorithm with integrated neural network. Li et al. [3] investigate the OP performance analysis and prediction. The OP expressions are derived in exact closed form. Zhou et al. [4] selected the multiple financial indicators based on big data mining in Internet of things. Yuan et al. [5] build a sports decisionmaking model based on data mining and neural network based on data mining technology and neural network algorithms. Lu et al. [6] introduce appropriate trend estimation methods, adjust pricing models and processes, and realize trend estimation that changes over time to make the resulting pricing model have advantages such as dynamics, accuracy, and flexibility over the original model. Xia et al.

Jinghua Zhao

zhaojinghua@usst.edu.cn

1 School of Business, University of Shanghai for Science and Technology, Shanghai 200093, China

2 School of Economics and Management, Changzhou Vocational Institute of Mechatronic Technology, 26 Mingxinzhong Road, Changzhou 213164, China
[7] carry out matrix-vector multiplication operations and weight update operations, design a perceptron neural network model, and realize a simulation platform based on MLP neural network. Liang [8] uses modern scientific and technological achievements and adopts scientific training methods and means to implement optimal control over the entire process of sports training. Xu et al. [9] aim to find the dynamic network traffic classification problem of hybrid fixed in dynamic network and dynamic network in mobile network and give a reasonable mapping scheme. Wang et al. [10] propose a fusion method of bilingual entity class named entity translation based on chunk symmetry strategy and English-Chinese transliteration model based on machine learning strategy.

Zhang and Jin [11] combine machine learning algorithms to improve the neural network and builds a credit system for the smart logistics public information platform. Zhou et al. [12] propose the cross-view similarity exploration (CVSE) method, which combines style-transferred samples to optimize the CNN model and the relationship between samples. Liu and Zheng [13] conduct research on the innovation process and innovation driving force based on autocatalytic network. Based on this, a remote monitoring platform for overflow accidents is proposed by Liang et al. [14]. Wu et al. [15] study the permeability characteristics of bedrock fissures based on the disturbance of neural network. Pang et al. [16] propose the farmers credit optimization decision model and applications based on common risk guarantee fund. TSAIB_RS method based on the two-stage adaptive integration of multi-source heterogeneous data was constructed by Huang and $\mathrm{Wu}$ [17]. Sun et al. [18] propose a mechanical adaptive control method based on a neural network. Hu et al. [19] study the classic reinforcement learning algorithm Q-learning and propose an improved Q-learning algorithm.

Acknowledgements The guest editors would like to thank Prof. John MacIntyre who is the editor in chief of Neural Computing and Applications. The guest editors would like to thank the reviewers for their high-quality reviews, which provided insightful and constructive 
feedback to the authors of the papers. The guest editors also would like to thank journal editor Rachel Moriarty, Deepika Sureshkumar, and Rashmi Jenna for their help on submission and publication.

\section{References}

1. Huang C, Zhu Z (2020) Complex communication application identification and private network mining technology under a large-scale network. Neural Comput Appl. https://doi.org/10. $1007 / \mathrm{s} 00521-020-05442-0$

2. Zhang L, Zhang L (2020) Top-N recommendation algorithm integrated neural network. Neural Comput Appl. https://doi.org/ $10.1007 / \mathrm{s} 00521-020-05452-\mathrm{y}$

3. Xu L, Wang H, Li H et al (2020) QoS intelligent prediction for mobile video networks: a GR approach. Neural Comput Appl. https://doi.org/10.1007/s00521-020-05441-1

4. Shang H, Lu D, Zhou Q (2020) Early warning of enterprise finance risk of big data mining in internet of things based on fuzzy association rules. Neural Comput Appl. https://doi.org/10. 1007/s00521-020-05510-5

5. Yuan C, Yang Y, Liu Y (2020) Sports decision-making model based on data mining and neural network. Neural Comput Appl. https://doi.org/10.1007/s00521-020-05445-x

6. Yu Y, Lu J, Shen D et al (2020) Research on real estate pricing methods based on data mining and machine learning. Neural Comput Appl. https://doi.org/10.1007/s00521-020-05469-3

7. Guo H, Guo C, Xu B et al (2020) MLP neural network-based regional logistics demand prediction. Neural Comput \& Applic. https://doi.org/10.1007/s00521-020-05488-0

8. Liang H (2021) Evaluation of fitness state of sports training based on self-organizing neural network. Neural Comput Appl. https:// doi.org/10.1007/s00521-020-05551-w

9. Liu D, Xu X, Liu M et al (2021) Dynamic traffic classification algorithm and simulation of energy Internet of things based on machine learning. Neural Comput Appl. https://doi.org/10.1007/ s00521-020-05457-7
10. Li P, Wang M, Wang J (2020) Named entity translation method based on machine translation lexicon. Neural Comput Appl. https://doi.org/10.1007/s00521-020-05509-y

11. Zhang H, Jin Y (2021) Credit system of smart logistics public information platform based on improved neural network. Neural Comput Appl. https://doi.org/10.1007/s00521-020-05547-6

12. Zhou S, Wang Y, Zhang F et al (2021) Cross-view similarity exploration for unsupervised cross-domain person re-identification. Neural Comput Appl. https://doi.org/10.1007/s00521-02005566-3

13. Liu C, Zheng X (2020) Exploring resource management for innovation power network based on deep learning algorithm. Neural Comput Appl. https://doi.org/10.1007/s00521-020-05446$\mathrm{w}$

14. Liang H, Han H, Ni P et al (2020) Overflow warning and remote monitoring technology based on improved random forest. Neural Comput Appl. https://doi.org/10.1007/s00521-020-05425-1

15. Zhang YZ, Wu X, Zhang X et al (2021) Permeability characteristics of bedrock fissures under disturbance conditions based on neural network. Neural Comput Appl. https://doi.org/10.1007/ s00521-020-05625-9

16. Pang S, Wei M, Wen Z (2021) Farmers credit optimization decision model and applications based on common risk guarantee fund. Neural Comput Appl. https://doi.org/10.1007/s00521-02005651-7

17. Huang A, Wu F (2020) Two-stage adaptive integration of multisource heterogeneous data based on an improved random subspace and prediction of default risk of microcredit. Neural Comput Appl. https://doi.org/10.1007/s00521-020-05489-z

18. Liu A, Zhao H, Song T et al (2020) Adaptive control of manipulator based on neural network. Neural Comput Appl. https://doi. org/10.1007/s00521-020-05515-0

19. Cui F, Hu H, Xie Y (2021) An intelligent optimization method of E-commerce product marketing. Neural Comput Appl. https:// doi.org/10.1007/s00521-020-05548-5

Publisher's Note Springer Nature remains neutral with regard to jurisdictional claims in published maps and institutional affiliations. 